\title{
Post-Pericardiotomy Syndrome
}

National Cancer Institute

\section{Source}

National Cancer Institute. Post-Pericardiotomy Syndrome. NCI Thesaurus. Code C111649.

An inflammatory disorder of the pericardium and pleura seen as a post-operative complication of cardiovascular surgery. 